\title{
L'apport genevois à l'hygrométrie
}

Par Margarida Archinard

L'Hygrométrie, c'est-à-dire, la mesure de l'humidité de l'air, est une science dont l'évolution fut particulièrement lente. Une des causes de cette lenteur provient, sans doute, du fait qu'il y a deux notions d'humidité à considérer.

L'une, l'humidité absolue, la première qui attira l'attention des savants et la monopolisa pendant longtemps, se définit comme étant la quantité de vapeur d'eau contenue dans une unité de volume d'air.

L'autre, l'humidité relative ou état hygrométrique de l'air, plus élaborée et par conséquent plus tardive, s'exprime par la fraction $r$

où

$$
\mathrm{E}=\frac{\mathrm{m}}{\mathrm{M}}
$$

m - est la masse de la vapeur d'eau existant dans un certain volume d'air,

M - la masse de vapeur d'eau qui existerait dans ce même volume d'air, à la même température, s'il était saturé, c'est-à-dire, s'il ne pouvait pas en contenir davantage.

Comme nous le voyons, l'humidité relative dépend de l'humidité absolue et de la température de l'air. C'est cette humidité relative, ou état hygrométrique de l'air, qui agit sur certaines substances du monde végétal et animal et qui est perceptible par nos propres sensations.

La prise de conscience de la présence de la vapeur d'eau dans l'air remonte à l'Antiquité. Les anciens Grecs et Hébreux, par exemple, avaient déjà une idée assez claire du cycle de l'eau. Dioscoride, si on en croit Porta, aurait même conseillé de suspendre des toisons de laine autour des navires pour se procurer de l'eau douce.

Mais il est difficile de préciser quand commença à être connue la capacité qu'ont certaines substances, dites hygroscopiques, de changer de poids et de volume avec l'état hygrométrique de l'air.

Tout le monde sait, pourtant, comment en 1586 l'architecte Domenico Fontana supplanta les insuffisances de l'échaffaudage destiné à dresser l'obélisque égyptien sur la place St. Pierre, à Rome, en mouillant les cordes.

Les premiers appareils conçus pour détecter, à défaut de pouvoir mesurer, les variations de l'humidité étaient basés sur cette propriété des substances hygroscopiques. On les appelle communément des hygromètres, mais ils ne furent, et pendant longtemps, que des hygroscopes. 
Le tout premier hygromètre fut inventé en 1450 par le Cardinal Nicolas de Cuse et consistait en une balance qui mesurait le changement du poids de quelques fils de laine avec l'humidité. Leonardo da Vinci le suivra dans cette voie.

Par la suite, les $\mathrm{XVI}^{\mathrm{e}}$ et $\mathrm{XVII}^{\mathrm{e}}$ siècles apportent une grande variété de ces instruments. Parmi les plus remarquables citons l'hygromètre à boyau du médecin vénicien Santorio Santorio (1561-1636); le «Mostra Umidaria» de Ferdinand II (1610-1670), Grand Duc de Toscane, qui, faisant exception à son époque, repose déjà sur l'observation de la condensation de la vapeur d'eau; et l'hygromètre d'Amontons (1663-1705) à réservoir en peau de mouton rempli de mercure qui transmettait ses variations de volume à une colonne d'esprit de vin surmonté d'huile.

Au XVIII ${ }^{\mathrm{e}}$ siècle, Charles Le Roy (1726-1779), professeur de Médecine à Montpellier, remarque enfin que l'humidité dépend de la température de l'air. Dans son mémoire, publié en 1751 par l'Académie Royale des Sciences de Paris, Le Roy avance même une notion nouvelle, celle du degré ou point de saturation qu'il traduit par «... un certain degré de froid auquel l'air est prêt à lâcher une partie de l'eau qu'il tient en dissolution» $\left({ }^{12}\right.$, p. 490$)$.

Et il complète son explication plus loin :

«Ces mots [air sec ou air humide] ne peuvent signifier, comme on le croit ordinairement, la quantité d'eau absolue que l'air contient ; ils doivent seulement désigner la quantité d'eau qu'il contient relativement à la chaleur. L'air peut être très desséchant un jour d'été, \& contenir beaucoup plus d'eau que l'air très humide d'un jour d'hiver. Dans une forte gelée par un vent de nord, l'air peut être plus éloigné du point de saturation (\& par conséquent plus desséchant) que l'air fort chaud d'un jour d'été» (12, p. 494-495).

Pour calculer le point de saturation, Le Roy fait varier la température d'un vase de cristal, par étapes d'un demi degré Réaumur, jusqu'à ce que la rosée apparaisse (ou disparaisse) sur sa paroi extérieure. Il jette ainsi, sans s'en douter, les bases des futurs hygromètres à condensation, les premiers capables de donner directement l'humidité relative.

Ils avaient, pourtant, un ancêtre dans le «Mostra Umidaria» de Ferdinand II qui était déjà vieux d'un siècle au moment où Le Roy rend publique son importante théorie sur le point de saturation. Mais dans la chaîne conduisant au premier hygromètre à condensation utilisable il manque encore un maillon, un seul mais de la plus haute importance: la notion même d'humidité relative. Elle fera enfin surface à l'aube du $\mathrm{XIX}^{\mathrm{e}}$ siècle et sera suivie de près par ce premier hygromètre à condensation qui s'est tant fait attendre.

Mais n'anticipons pas. 
C'est entre-temps qu'interviennent les travaux des trois illustres savants genevois : Jean André De Luc, Jean Senebier et Horace Bénédict de Saussure.

Voyons en quoi ils ont consisté.

Le premier des trois qui s'occupa d'hygrométrie fut Jean André De Luc (1727 à 1817). Né à Genève, ses opinions politiques l'obligent, en 1773, à s'expatrier à Londres où il a vécu jusqu'à sa mort. Déjà célèbre par son baromètre portatif et sa formule barométrique ${ }^{1}$, exposés dans son grand ouvrage Recherches sur les modifications de l'Atmosphère ${ }^{4}$, il devient membre de la Royal Society et Lecteur de la Reine dès son arrivée en Angleterre. Peu après, en octobre 1773, il présente à la Royal Society un mémoire sur un hygromètre de son invention, qui fut couronné par l'Académie d'Amiens en 1774.

Les toutes premières lignes de ce mémoire nous font connaître ses motivations et son état d'esprit :

«En augmentant ainsi le désir d'un hygromètre, j'ai craint longtems d'augmenter seulement le regret de n'en point avoir. Ce regret, que j'éprouvois déjà moimême, étant devenu chez moi une sorte d'inquiétude, qui me suivoit dans toutes mes occupations, j'ai franchi enfin des obstacles que je croyois insurmontables: j'ai imaginé un hygromètre et je l'ai exécuté» $\left({ }^{5}\right.$, p. 381).

L'hygromètre qu'imagina De Luc était basé sur les propriétés hygroscopiques de l'ivoire car, dit-il, «je me rappellai que la clef d'un robinet d'ivoire dont j'avois fait usage [probablement celui de son baromètre portatif], tournoit avec plus ou moins de peine, suivant que l'air étoit plus ou moins humide» $\left({ }^{5}\right.$, p. 387$)$.

Il construit ainsi un récipient cylindrique en ivoire qu'il relie par une pièce en laiton, bien ajustée, à un tube de verre analogue à ceux utilisés dans ses thermomètres. Le tout est rempli de mercure de façon à ce que les variations de hauteur du mercure dans le tube de verre traduisent les variations de volume du cylindre d'ivoire avec l'humidité.

Ce procédé rappelle beaucoup celui de l'hygromètre d'Amontons. Mais De Luc apporte une innovation importante avec sa recherche d'une échelle adéquate à la mesure de l'humidité. Son premier souci est de trouver deux points fixes. Il choisit les points de sécheresse absolue et d'humidité extrême, selon sa propre terminologie, adoptée d'ailleurs par la suite par Senebier et Saussure. Mais il est obligé de se contenter de ce dernier et d'abandonner le point de sécheresse absolue, car «... n'ayant découvert aucun moyen d'y parvenir que par le feu, \& le feu ne la produisant dans tous les corps qui me parurent susceptibles d'humidité qu'en changeant leur nature, je vis avec bien de la peine que j'étois réduit à chercher mon point fixe, là précisément où j'avois le moins espéré de le découvrir. Je demeurai en effet assez long-tems sans rien appercevoir dans cette nouvelle route. Plusieurs fois je re- 
tournai en arrière, mais toujours je fus ramené à l'humidité extrême, comme à la seule face de mon objet qui me laissât quelque espérance de le saisir» ( ${ }^{5}$, p. 384).

De Luc fixe donc le zéro degré de son échelle au point d'humidité extrême. Influencé par les idées de Le Roy, il le définit en tenant compte de la température :

«Ce point... est celui où la sécheresse est nulle (s'il m'est permis de m'exprimer ainsi), puisque c'est celui de l'humidité extrême, par une chaleur donnée, qui est celle de la glace fondante : c'est de ce point que doivent se compter les degrés dont je vais parler, qui deviennent ainsi des degrés de dessèchement» $\left({ }^{5}\right.$, p. 395$)$.

Et il l'obtient en plongeant la pièce en ivoire de son hygromètre dans un vase rempli de glace fondante constamment renouvelée.

Le choix du degré de cette échelle présente quelques difficultés car De Luc ne dispose pas d'un deuxième point fixe. Mais il les résout en prenant pour base le degré d'un thermomètre de Réaumur, c'est-à-dire, d'un thermomètre divisé en 80 parties entre le point de fusion de la glace et le point d'ébullition de l'eau. Il fait le raisonnement suivant: «... l'étendue des degrés d'un hygromètre doit être à celle des degrés du thermomètre préparatoire, comme le poids du mercure de l'hygromètre est au poids du mercure que contenoit ce thermomètre» $\left({ }^{5}\right.$, p. 395$)$.

Et, en divisant cette «étendue des degrés d'un hygromètre», qu'il appelle plus loin ligne fondamentale, en 40 parties pour mieux faciliter la lecture, le degré de l'hygromètre de De Luc devient, tout compte fait, égal à presque $2 / 3$ du degré de son thermomètre.

Pour corriger l'effet de la chaleur sur le mercure et ramener toujours la température à $0^{\circ}$ Réaumur, De Luc grave son échelle sur une tringle mobile le long du tube de l'hygromètre.

A côté, est fixée une petite échelle dont le degré représente la $80^{\mathrm{e}}$ partie de la ligne fondamentale de l'hygromètre: «...par conséquent ils [les degrés de cette petite échelle] sont immédiatement correspondans aux degrés du thermomètre que porte la même monture» $\left({ }^{5}\right.$, p. 397$)$.

Ainsi, pour une certaine température de l'air, cette correction se fait en déplaçant l'échelle mobile de façon à ce que l'index qu'elle porte coïncide, sur la petite échelle fixe, avec le même nombre de degrés que celui de la température de l'air, donné par le thermomètre qui l'accompagne.

Pour étudier les possibilités et l'exactitude de son appareil, De Luc en fait construire quatre autres exemplaires et les soumet à plusieurs sortes d'expériences. Les résultats ne furent pas très encourageants et il reconnait «... qu'ils [ces appareils] ne conservent pas toujours les mêmes rapports entr'eux» $\left({ }^{5}\right.$, p. 459$)$. 
Quant à la graduation de son hygromètre, De Luc savait d'avance qu'elle ne donnerait pas la quantité réelle d'humidité, mais «l'essentiel étoit qu'on pût s'entendre lorsqu'on parleroit de degrés d'humidité» $\left({ }^{5}\right.$, p. 383).

La discussion n'est pourtant pas close. Les derniers mots de son mémoire sont :

«J'en ai dit assez pour montrer que cette matière est vaste, \& qu'elle mérite un examen attentif» $\left({ }^{5}\right.$, p. 476$)$.

Le deuxième Genevois à y porter un examen attentif fut Jean Senebier (1742 à 1809), pasteur, naturaliste et bibliothécaire de la Ville de Genève.

Il connaissait très bien l'œuvre de De Luc et la tenait en très haute estime. Sa critique de l'hygromètre de De Luc est riche en renseignements :

«Cet instrument est un chef-d'œuvre d'invention \& d'exécution, mais il a plusieurs défauts assez considérables; je ne veux pas les analyser ici, parce que l'Auteur Philosophe de cet instrument, les a trouvés assez considérables pour abandonner son invention, \& pour lui en substituer une autre que je ne connois pas assez bien pour en parler. Mais, en supposant l'hygromètre de M. de Luc excellent, il avoit un défaut essentiel dans la difficulté de l'exécution, qui la rendoit presque impossible à tout autre qu'à son Auteur, qui est aussi adroit \& patient, que plein de savoir \& de génie» $\left({ }^{22}\right.$, p. 427$)$.

Quant à ses propres travaux en hygrométrie, Senebier en fait état avec la plus grande modestie. En effet, on peut lire à la première page de son mémoire sur les hygromètres, publié en 1778 par les Observations sur la Physique:

«M. De Luc s'est élevé par son génie jusqu'à la partie la plus sublime de cette recherche, \& il a eu des succès que personne n'avoit eus avant lui. Je ne prétends pas à de si grandes choses \& je suis bien éloigné d'y prétendre» ( ${ }^{22}$, p. 421$)$.

En outre, Senebier ajoute à la fin de son mémoire une lettre de H. B. de Saussure où il est pour la première fois question d'un hygromètre basé sur les propriétés hygroscopiques du cheveu (il s'allonge quand l'humidité augmente et raccourcit quand l'humidité diminue). L'importance que prît cette lettre par la suite étouffa quelque peu le mérite des expériences de Senebier qui y sont relatées et qui ne manquent pas d'intérêt.

Pour ces expériences, Senebier emploie l'hygromètre de Lambert, composé d'une corde de boyau comme celui, bien plus ancien, de Santorio. Il lui apporte quelques perfectionnements mais c'est dans la recherche des deux points fixes, notamment dans la recherche du point de sécheresse absolue, qu'il s'illustre. En effet, nous avions vu que De Luc avait été contraint d'abandonner ce dernier car il ne trouvait pas d'autre moyen que le feu, moyen impraticable, pour y arriver. Or Senebier utilise les propriétés de certains sels «... qui attirent l'humidité; l'augmentation de leur poids fait connaître la quantité d'humidité qu'il y a dans l'air» 
$\left({ }^{22}\right.$, p. 429). Il énumère plusieurs de ces sels mais emploie surtout le sel de tartre. «Par ce sel de tartre, j'entends celui qui porte le nom d'alkali purifié» ( ${ }^{22}$, p. 431$)$.

Ainsi, pour obtenir le point de sécheresse absolue, Senebier enferme dans un vase clos son hygromètre à boyau et une quantité de sel de tartre suffisante pour absorber toute la vapeur d'eau à l'intérieur du vase.

Pour le point d'humidité extrême, Senebier mouille d'abord la corde, comme De Luc, dans une eau «... qui commence à se geler» $\left({ }^{22}\right.$, p. 434$)$, la fait sécher et ensuite l'enferme dans un vase de verre où il fait évaporer de l'eau jusqu'à saturation.

Sur un cadran, une aiguille couvrira un demi cercle environ pour passer d'un point fixe à l'autre.

Saussure utilise à peu de choses près le même procédé pour la graduation de son hygromètre à cheveu. L'a-t-il appris de Senebier ou l'a-t-il trouvé indépendamment, la question pourrait se poser. Mais elle est secondaire. Le succès de l'hygromètre de Saussure fut si retentissant qu'il marque une époque incontestable dans l'histoire de l'hygrométrie.

Horace Bénédict de Saussure (1740-1799), le plus connu des savants genevois, est-il necéssaire de le présenter aux lecteurs ? Elu professeur de Philosophie à l'Académie de Genève en 1762, son esprit étonnamment polyvalent, et toujours en éveil, l'incite à s'intéresser et à s'occuper de différentes questions scientifiques. Il y met tant d'enthousiasme et d'adresse qu'il devient célèbre aussi bien en Géologie, avec ses Voyages dans les Alpes et ses conclusions quant à leur formation, qu'en Botanique, avec ses Observations sur l'écorce des feuilles et des pétales, et en Hygrométrie, avec son fameux hygromètre à cheveu.

D'autres inventions, comme celle, pour le moins inattendue, de deux appareils destinés l'un à mesurer l'intensité de la couleur bleue du ciel ${ }^{20}$, l'autre la transparence de l'air ${ }^{21}$, témoignent d'un dynamisme d'autant plus remarquable qu'il est aux prises avec de sérieux ennuis de santé depuis sa $30^{\mathrm{e}}$ année.

Ses publications furent très nombreuses mais nous ne nous occuperons ici que de celles concernant l'hygrométrie.

La première date donc de 1778 et a la forme d'une lettre adressée à Jean Senebier, comme nous venons de voir. Saussure y décrit le premier hygromètre à cheveu qu'il a conçu et ses expériences pour juger de ses qualités.

Cet hygromètre était formé par un cheveu, préalablement bouilli dans une lessive contenant une faible quantité d'alkali caustique (10 ou 12 grains pour 5 ou 6 onces d'eau), qu'on disposait verticalement, accroché par une extrémité à un point fixe et enroulé, par l'autre extrémité, «... autour d'un petit cylindre armé d'une aiguille légère, qui marquoit ses révolutions sur un cadran» $\left({ }^{15}\right.$, p. $\left.435-436\right)$. Un contre- 
poids de 12 grains $(0,6 \mathrm{~g})$, suspendu à un fil de soie enroulé autour du même cylindre dans le sens contraire du cheveu, maintenait le cheveu toujours tendu.

Peu de détails seront changés par la suite à ce premier essai mais la conclusion de Saussure était alors plutôt pessimiste: «...des expériences répétées m’ont fait découvrir dans le cheveu une propriété qui, à moins qu'on ne trouve le moyen d'y remédier, le rend tout-à-fait impropre à servir de matière à un hygromètre durable. Lorsqu'il est pendant long-tems exposé à un air sec, il s'affoiblit \& s'allonge peu-à-peu, \& lorsqu'ensuite on le replace dans un air humide, l'humidité le renforce \& le contracte de nouveau à un tel point, que si on le rapporte alors dans le même air sec dont il a été tiré, cette contraction lui fait indiquer une sécheresse plus grande qu'il n'indiquoit auparavant» $\left({ }^{15}\right.$, p. 438$)$.

Cependant, il ne perd pas espoir car il ajoute plus loin : «Si un Physicien plus habile ou plus heureux que moi, parvient à remédier à cet inconvénient, le cheveu donnera sûrement le meilleur de tous les hygromètres» $\left({ }^{15}\right.$, p. 438$)$.

Et il eut raison de persister dans son idée et de continuer ses recherches car cinq ans plus tard, en 1783, la publication de son important ouvrage Essais sur l'Hygrométrie impose enfin, et pour longtemps, l'hygromètre à cheveu.

Saussure y propose même deux sortes d'appareils, l'un qu'il nomme grand hygromètre ou hygromètre à arbre, destiné à rester sédentaire dans un laboratoire, l'autre, plus léger et conçu pour supporter le transport, qu'il appelle simplement hygromètre portatif.

Pour pallier au défaut du cheveu, auquel il fait allusion dans sa lettre à Sénebier en 1778 , il réduit le contrepoids, destiné à le tendre, à 3 grains $(0,16 \mathrm{~g})$.

«Une autre précaution que j'ai cru devoir employer, c'est d'arrêter les cheveux par des pinces à vis, au lieu de les nouer, comme je faisois autrefois» $\left({ }^{16}, \S 16\right)$.

Par ailleurs, Saussure ne les roule plus autour d'un cylindre, car «...il [le cheveu] se frisoit \& contractoit une roideur que le contre-poids ne pouvoit point surmonter» $\left({ }^{16}, \S 2\right)$.

Ainsi, dans l'hygromètre à arbre, le cheveu est lié par une pince à une fine lame d'argent qui, elle, s'enroule autour du cylindre porteur de l'aiguille destinée à indiquer sur un cadran les variations de longueur du cheveu avec l'humidité. Dans l'hygromètre portatif, il n'y a point de cylindre semblable mais un dispositif assez ingénieux porté par l'aiguille elle-même. Laissons Saussure l'expliquer :

«La partie... de l'aiguille sert d'indice \& marque sur le cadran les degrés d'humidité et de sécheresse ; la partie opposée... sert à fixer \& le cheveu \& le contre-poids. Cette partie qui se termine en portion de cercle... est creusée sur son champ d'une double rainure verticale, qui rend cette partie semblable à un segment d'une poulie à double gorge. Ces deux rainures qui sont des portions d'un cercle... dont 
le centre est le même que celui de l'aiguille... servent à loger, l'une le cheveu, \& l'autre la soie à l'extrêmité de laquelle est suspendu le contre-poids. Cette même aiguille porte verticalement au-dessus \& au-dessous de son centre, deux petites pinces à vis, situées vis-à-vis des deux rainures; celle d'en-haut... vis-à-vis de la rainure postérieure, sert à fixer la soie à laquelle est suspendu le contre-poids... \& celle d'en-bas... située vis-à-vis de la rainure antérieure, sert à tenir une des extrêmités du cheveu» $\left({ }^{16}, \S 4\right)$.

Ces perfectionnements au projet initial de Saussure purent être réalisés grâce à l'active collaboration de l'excellent artisan genevois Jaques Paul à qui, d'ailleurs, De Luc avait déjà confié l'exécution de ses baromètres.

Saussure en parle en ces termes fort élogieux : «M. Paul, l'un des Artistes les plus distingués de notre ville, \& qui est capable, non seulement d'exécuter les instrumens les plus délicats, mais de perfectionner même les idées du Physicien qui les fait construire, a fait pour moi un grand Hygromètre à arbre \& plusieurs portatifs, qui ont toute la perfection dont leur construction les rend susceptibles » $\left({ }^{16}\right.$, $\S 4$, note).

Et Daumas, de nos jours, ajoutera: «L'inventeur et le constructeur ont une part égale dans le succès de cet instrument. En effet, si sa conception en est simple, et si les données de son fonctionnement ont été précisées avec soin, il est d'une construction solide et pratique pour l'époque. Le fait que les proportions et la disposition convenables, les petits détails de construction qui lui donnent ses qualités et son fini, aient été trouvés dès les premiers essais, montre assez bien quel était alors le niveau technique des meilleurs fabricants de ce petit appareillage de physique. Un siècle plus tôt, un problème de cet ordre devait passer par plusieurs ateliers avant de connaître une solution satisfaisante» $\left({ }^{3}\right.$, p. 284) .

Mais d'autres précautions s'étendent encore à la préparation et au choix du cheveu.

Pour les dégraisser, Saussure abandonne l'alkali caustique, jugé trop puissant, et utilise à sa place le sel de soude crystallisé (carbonate de soude) dans les proportions suivantes : «... si l'on prend de l'eau pure \& qu'on y ajoute six grains de ce sel par once, cette lessive a un degré de force tel, qu'en vingt-cinq ou trente minutes d'ébullition, elle donne aux cheveux toute la mobilité que l'on peut souhaiter» $\left({ }^{16}, \S 8\right)$.

Il se contentait aussi auparavant de lier les cheveux avant de les faire bouillir, maintenant il les enveloppe dans une bande de toile et les y coud comme dans un sac.

Quant à la graduation de ses hygromètres, Saussure recherche, comme ses prédécesseurs, les points de sécheresse absolue et d'humidité extrême et utilise pour cela une méthode très semblable à celle de Senebier décrite plus haut. Comme lui, 
pour le point d'humidité extrême, Saussure fait évaporer de l'eau dans une cloche de verre hermétique mais sans mouiller au préalable sa substance hygroscopique. Comme lui encore, pour le point de sécheresse absolue, Saussure emploie un sel absorbant fortement la vapeur d'eau mais, après avoir essayé le sel de tartre, il lui préfère le carbonate de potasse. Il le prépare d'ailleurs lui-même en aspergeant «... d'une poudre composé de parties égales de nitre \& de tartre crud» $\left({ }^{16}, \S 21\right)$ les deux côtés d'une feuille de tôle, en forme de demi-cylindre et chauffée au rouge avant et après l'opération, qu'il introduit encore assez chaude dans la cloche de verre où se trouve son hygromètre.

Et pour être sûr d'avoir vraiment atteint le point de sécheresse absolue, Saussure expose le tout à une source de chaleur telle que le Soleil, par exemple. Le cheveu commence par se dilater, mais s'il existe encore de la vapeur d'eau dans la cloche, le cheveu se contracte à nouveau et l'aiguille s'arrête à un point correspondant à cette humidité. Si, par contre, l'opération a été réussie, si en effet le point de sécheresse absolue a été atteint, le cheveu ne fait qu'augmenter de volume avec la chaleur et l'hygromètre, à ce moment, se comporte comme un thermomètre. «L'allongement du cheveu par la chaleur est donc le critère du desséchement parfait» $\left({ }^{16}, \S 23\right)$.

Saussure fixe alors le $0^{\circ}$ de son échelle à ce point de sécheresse absolue, qui est visiblement indépendant de la température, et attribue les $100^{\circ}$ au point d'humidité extrême sans tenir compte, au contraire de De Luc, de la température. Il dira même: "On ne doit donc point craindre que la chaleur plus ou moins grande, soit de l'eau, soit des vapeurs, soit de l'air ambiant, produise un changement sensible sur le terme de l'humidité extrême» $\left({ }^{16}, \S 18\right)$, ce qui deviendra un des points litigieux de la dispute qui suivra entre Saussure et De Luc, comme on le verra plus loin. Mais revenons à l'échelle établie par Saussure sur ses hygromètres. Cette échelle, qui a déjà l'avantage d'être centésimale (la distance entre les deux points fixes est donc divisée en 100 parties égales), Saussure l'applique différemment dans son hygromètre à arbre et dans l'hygromètre portatif. Dans ce dernier, l'échelle est gravée directement sur le cadran qui a la forme d'un arc de cercle. Dans l'hygromètre à arbre, le cadran, qui est un cercle entier, garde sa division classique en $360^{\circ}$ mais est accompagné d'une table indiquant à quels degrés du cercle correspondent les degrés de l'échelle hygrométrique en 100 parties. Par ailleurs, comme chaque échelle n'est valable que pour le cheveu avec lequel on l'a déterminée, l'hygromètre portatif a aussi besoin d'une table de conversion quand, pour une raison quelconque, on est obligé d'en changer le cheveu.

L'hygromètre à cheveu est maintenant prêt à fonctionner et Saussure va le soumettre à une longue et très complète série d'expériences. 
Sachant déjà qu'«un cheveu lessivé à propos, en passant de la sécheresse extrême à l'humidité extrême, s'allonge de 0,0245 de sa longueur totale» $\left({ }^{16}, \S 28\right)$, Saussure cherche encore à connaître l'action de la chaleur directement sur le cheveu, question déjà soulevée au moment de la détermination du point de sécheresse absolue. Il conclut qu'«... un degré de chaleur [ $1^{\circ}$ Réaumur] allonge le cheveu d'une quantité qui équivaut à-peu-près à la treizième partie d'un degré de l'échelle hygrométrique, quantité que l'on peut négliger dans les observations courantes, \& dont on pourroit aisément tenir compte dans les observations délicates \& importantes, si du moins il est permis de supposer que la chaleur dilate toujours le cheveu suivant la même loi, dans les divers degrés d'humidité dont il est susceptible» $\left({ }^{16}, \S 28\right)$.

Dans cette ordre d'idées, Saussure dresse une table des différentes variations infligées par un changement de température de $1^{\circ}$ Réaumur sur l'hygromètre à cheveu, en fonction du degré d'humidité qu'il indique. On y remarque que plus ce degré d'humidité est élevé (la table le donne à partir de $25^{\circ}$ jusqu'à $100^{\circ}$ ), plus la variation apportée par le degré Réaumur est grande.

Une deuxième table complémentaire établit, aussi pour chaque degré d'humidité (de $25^{\circ}$ à $99^{\circ}$ ), les augmentations ou diminutions de température nécessaires pour faire varier l'hygromètre d'un degré et le nombre de degrés de refroidissement nécessaires pour l'amener au point de saturation.

«Ces tables ont encore l'avantage de dispenser de toute correction pour les dilatations \& contractions pyrométriques du cheveu» $\left({ }^{16}, \S 93\right)$, dit Saussure, et en effet elles sont utiles pour comparer plusieurs observations faites à des températures différentes.

D'autres expériences vont mener Saussure à chercher encore l'effet de l'agitation et de la raréfaction de l'air ainsi que de l'électricité sur son hygromètre à cheveu.

Mais une question majeure l'arrête plus longtemps, c'est le rapport existant entre les degrés de l'hygromètre et la quantité de vapeur d'eau contenue dans l'air.

Saussure propose alors une table grâce à laquelle «... l'hygrométrie seroit alors portée au plus haut point de perfection qu'elle puisse atteindre» $\left({ }^{16}, \S 180\right)$ et qui donnerait, pour chaque pouce du baromètre et pour chaque degré du thermomètre et de l'hygromètre, le poids de la vapeur d'eau contenue dans un pied cube d'air. Mais il reconnait l'immensité du travail et l'impossibilité, pour lui, d'y consacrer tout le temps qu'il faudrait. Cependant, en remplacement de cette table, disons, idéale, Saussure en présente une autre de plus petites dimensions et obtenue par une méthode indirecte, donc moins sûre, mais plus rapide. Elle est établie pour une pression atmosphérique de 27 pouces (une autre table l'accom- 
pagne avec les coefficients de conversion à d'autres pressions, comptées tous les $3^{1 / 8}$ pouces depuis 27 pouces jusqu'à $2 \frac{1}{2}$ lignes). Les degrés du thermomètre (de $-10^{\circ}$ à $+30^{\circ}$ Réaumur) ainsi que ceux de $1^{\prime}$ hygromètre (de $40^{\circ}$ à $95^{\circ}$ et même $98^{\circ}$ ) ne rentrent dans la table que de $5^{\circ}$ en $5^{\circ}$.

L'étendue et la qualité de ces expériences feront dire aujourd'hui à Middleton : «De Saussure was indeed an experimenter of great distinction, even in comparison with Lavoisier, Priestley, and Cavendish» $\left({ }^{13}\right.$, p. 106). Et à Daumas : «Les travaux de Saussure étaient assez précis et complets pour accréditer son instrument dont les indications furent considérées jusqu'à Regnault comme constantes» $\left({ }^{3}\right.$, p. 283-284).

Mais, comme c'est souvent le cas, Saussure a quand même trouvé quelques objections de la part d'autres physiciens dont notamment De Luc.

Expatrié toujours à Londres, De Luc reçoit, par l'intermédiaire de M. A. Pictet, un hygromètre de Saussure avec lequel, et en comparaison avec le sien, il va procéder à plusieurs essais.

Mais l'hygromètre de De Luc était à ce moment-là à sa troisième version. De la première, qui date de 1773 et que nous avons longuement décrite plus haut, son inventeur avouera : «... je découvris bientôt après que l'ivoire (qui étoit sa substance hygroscopique) n'avoit point toujours la même dilatabilité» $\left({ }^{6}, \S 35\right)$. Et il abandonne alors l'ivoire pour le fanon de baleine. Nous avons peu de renseignements sur ce deuxième hygromètre. Il fut présenté à l'Académie des Sciences de Paris en 1781 et, comme le premier, ne possédait qu'un seul point fixe, le point d'humidité extrême.

Saussure en parle en ces termes : «M. Deluc, à qui j'avois fait voir ces Hygromètres [à cheveu] il y a plusieurs années, vient d'adapter le même méchanisme à des lames très minces de fanon de baleine, dont il croit pouvoir faire de bons hygromètres. Le seul changement de quelqu'importance qu'il ait fait à cette construction, c'est d'employer un ressort au lieu d'un poids pour tenir son ruban tendu» $\left({ }^{16}, \S 3\right.$, note) .

Et De Luc s'explique : «Je préfère les ressorts aux poids pour tenir ces bandelettes tendues, non-seulement parce que les premiers sont plus commodes dans le transport, mais sur-tout parce qu'ils tiennent la bandelette constamment tendue; ce qui est très essentiel» $\left({ }^{6}, \S 58\right)$. Et il poursuit : «Les ressorts ont encore un avantage sur les poids, \& la baleine sur nombre d'autres substances, quand l'hygromètre est observé au vent. Le vent agite les poids, \& rend l'indication de l'instrument fort incertaine» $\left({ }^{6}, \S 59\right)$.

La troisième et dernière version de son hygromètre est enfin munie des deux points fixes d'humidité et de sécheresse extrême (le premier obtenu toujours par 
immersion dans l'eau, le second en vase clos avec de la chaux) et d'une échelle centésimale analogue à celle de Saussure. C'est cet appareil que De Luc utilisera comme terme de comparaison pour étudier à son tour la marche de l'hygromètre de Saussure qu'il avait reçu de M. A. Pictet. Regrettant de ne disposer que d'un seul de ces hygromètres à cheveu, il empruntera encore celui de George Adams pour ses expériences (remarquons au passage que Saussure dit que Paul en a construit environ 150, dont la plupart serait passée par ses mains; il n'est donc pas étonnant que d'autres savants à l'étranger en possèdent aussi).

Les conclusions de De Luc, qui enserrent une critique de l'œuvre de Saussure qui ne sera pas du goût de ce dernier, paraissent en 1786 dans son ouvrage Idées sur la Météorologie.

Tout en reconnaissant que le livre de Saussure, Essais sur l'Hygrométrie, est «... rempli de faits intéressans \& de remarques que personne n'avoit encore publiées» $\left({ }^{6}, \S 38\right)$, De Luc affirme quand même son désaccord avec plusieurs points de ce travail.

Le plus important de ces points de discorde, en tout cas celui qui les sépara davantage, fut l'emploi par Saussure d'une cloche de verre, hermétique et constamment humectée, pour l'obtention du point d'humidité extrême.

Partisan convaincu du mouillage intégral de la substance hygroscopique, De Luc déclare: «Je conclus donc; comme je l'avois fait en 1773 dans mon premier ouvrage sur l'Hygrométrie ; que c'est dans l'eau qu'on trouve sûrement l'humidité extrême ; \& j'ajoute maintenant, qu'elle s'y trouve à toute température [rappelons qu'auparavant De Luc la calculait pour $0^{\circ}$ Réaumur]. On la trouve aussi dans le brouillard; mais c'est seulement, parce qu'il couvre d'eau la substance de l'hygromètre. ... Il n'est donc rien d'aussi sûr, comme de plus simple, que de plonger l'hygromètre dans l'eau, pour fixer son point d'humidité extrême» $\left({ }^{6}, \S 49\right)$.

Cependant, il comprend pourquoi Saussure «... craignant de plonger son hygromètre dans l'eau, à cause de sa construction, a rejetté ce moyen, comme n'étant pas convenable» $\left({ }^{6}, \S 38\right)$. Mais il continue à croire que «...vu la complication des causes qui agissent dans les vases clos» $\left({ }^{6}, \S 39\right)$, le point d'humidité extrême y varie avec la température et même à une température «en apparence constante» $\left({ }^{6}, \S 39\right)$.

Et, comme preuve, De Luc avance le résultat de ses expériences faites sous la cloche humectée. Les changements de température au cours de la journée y entraînent d'importantes variations dans son hygromètre à fanon de baleine (pas toujours dans le même sens, il est vrai, et Saussure ne manquera pas de le faire remarquer) tandis que l'hygromètre à cheveu y reste pratiquement immuable. 
Par contre, à l'air libre et dans le brouillard, l'hygromètre de De Luc se maintient sensiblement autour des $100^{\circ}$ mais les deux hygromètres de Saussure (l'un à lui, De Luc, l'autre à George Adams) varient entre $96^{\circ}$ et $99^{\circ}$. Une fois même, un de ces hygromètres à cheveu, celui appartenant à De Luc, a baissé dans le brouillard jusqu'à $90^{\circ}$. De Luc est, pourtant, le premier à s'en étonner : "Il semble donc que ce cheveu aît perdu de son expansibilité dans le cours de mes expériences; car je ne lui apperçois aucune autre cause de dérangement» $\left({ }^{6}, \S 78\right)$. Et Saussure ajoutera plus tard, un peu agacé : «En effet, il est bien naturel que toutes les tortures que M. De Luc a fait subir à cet instrument l'ayent enfin dérangé» $\left({ }^{19}\right.$, p. 31).

Une autre question débattue entre les deux Genevois fut la rétrogradation, c'est-à-dire, «... ce défaut de certains cheveux, qui dans l'humidité extrême, commencent par s'allonger pour se raccourcir ensuite» $\left({ }^{19}\right.$, p. 30$)$.

De Luc prétend que «... M. de Saussure, en rejettant tous les cheveux dont la rétrogradation excède une certaine petite quantité, prépare l'accord de ses propres hygromètres» $\left({ }^{6}, \S 96\right)$.

Ce à quoi Saussure réplique: «J'ai si peu dissimulé ce défaut, que je lui ai moi-même donné le nom qu'il porte. J'ai même indiqué le moyen de le reconnaître, \& j'ai soigneusement averti de mettre au rebut les cheveux qui rétrograderaient de plus d'un ou deux degrés. Il paroit que l'on a négligé ce soin dans les deux hygromètres qu'a observés M. De Luc puisque leur rétrogradation est de 4 degrés » $\left({ }^{19}\right.$, p. 30$)$.

Les critiques de De Luc s'étendent encore à d'autres détails, comme par exemple la théorie de Saussure «... dans laquelle il regarde l'air comme un dissolvant de l'eau » $\left({ }^{6}, \S 85\right)$, mais ils sont de moindre importance.

Saussure prendra très mal les remarques de De Luc. Il faut dire que De Luc, de 13 ans l'aîné de Saussure et jouissant d'une solide réputation dans les milieux scientifiques, considérait Saussure au départ quelque peu comme un amateur. Nous pouvons lire déjà dans ses Recherches sur les modifications de l'atmosphère, qui datent de 1772, cette phrase assez significative : «M. Desaussure, Professeur en Philosophie à Genève, \& qui cultive par goût les sciences relatives à sa profession, ...» $\left({ }^{4}, \S 643\right)$.

Par ailleurs, contraint d'abandonner l'ivoire et après avoir essayé les os, les plumes et bien d'autres substances hygroscopiques, De Luc vient enfin d'adopter le fanon de baleine pour son hygromètre quand justement sort le remarquable ouvrage de Saussure prônant, expériences et tables à l'appui, une substance si commune et si simple, le cheveu. La surprise de De Luc a dû être considérable bien que pas un instant il l'ait laissé transparaître. Il reconnaît, cependant, que 
«M. de Saussure a eu sur moi un grand avantage à cet égard (si c'est un que de trouver trop de facilité dans sa première route, \& d'être dispensé par-là de regarder à chaque pas autour de son objet). La nature lui a fourni dans le cheveu un fil hygroscopique tout préparé: au lieu que je n'ai pu obtenir des fils de baleine en travers, que par une graduation très-lente. Mais enfin j’y suis parvenu » $\left({ }^{8}\right.$, p. 135$)$.

Les observations de De Luc sur l'hygromètre à cheveu, contenues dans ses Idées sur la Météorologie, dont le ton est toujours très correct, peuvent quand même sembler un peu péremptoires par moments. Saussure, en tout cas, a dû les ressentir comme telles. Pourtant, le découragement de De Luc est alors certain. Il y avoue même : «... je médite ma retraite... quand on a 60 ans \& des devoirs à remplir, si on se trouve encore à la suite de recherches intéressantes qui exigent ces facultés, il est tems d'en remettre le fil à d'autres» $\left({ }^{6}, \S 449\right)$.

La riposte de Saussure ne se fît pas attendre. Il rédige, au cours de l'année qui suit, un long article intitulé Défense de l'hygromètre à cheveu qu'il envoie en France au journal de l'Abbé Rozier, Observations sur la Physique, et en Angleterre à Sir Joseph Banks, président de la Royal Society. Pour finir, il le fait encore publier à son compte à Genève, en 1788.

La plus grande partie de son texte est consacrée à De Luc mais deux autres physiciens y sont aussi concernés. Ce sont Chiminello, astronome attaché à l'Observatoire de Padoue, et le Père Jean-Baptiste, capucin du couvent de St. Martin à Vicence.

C'est convaincu de la mauvaise foi de ses opposants que Saussure commence sa Défense...: "Trois physiciens se sont élevés contre cet ouvrage [Essais sur l'Hygrométrie ]; mais plus encore contre l'instrument que contre la théorie : \& ce qu'il y a de bien remarquable, c'est que chaqu'un de ces trois physiciens est inventeur d'un hygromètre différent du mien; \& que chacun d'eux n'a déprimé mon hygromètre que pour exalter le sien» $\left({ }^{19}\right.$, p. 2$)$.

Quant aux remarques de De Luc touchant à son point d'humidité extrême, Saussure conviendra: "C'est donc le $98^{\mathrm{e}}$. degré de mon hygromètre qui indique le vrai point de saturation de l'air; les deux derniers degrés dont il s'élève, lorsqu'il est plongé dans un air supersaturé, ne sont qu'une extention méchanique produite par cette eau surabondante» $\left({ }^{19}\right.$, p. 17). Mais il maintient l'usage de la cloche de verre pour sa détermination et désapprouve l'opinion de De Luc qui «... croit que sous une cloche constamment humectée la chaleur doit faire marcher l'hygromètre à la sécheresse» $\left({ }^{19}\right.$, p. 7$)$, car «... quand on voit ces instrumens si mobiles, si sensibles, inébranlables autour du même degré, malgré des changemens considérables dans la température de la cloche, peut-il rester encore quelque doute ?» $\left({ }^{19}\right.$, p. $\left.13-14\right)$. 
Saussure aurait dû s'en tenir là car sa cause a déjà de bons arguments pour sa défense. Mais il va plus loin, il attaque De Luc de manière parfois insultante comme en témoignent ces quelques phrases : «... M. De Luc, dont les hypothèses résistent rarement à des épreuves trop sévères, ...» $\left({ }^{19}\right.$, p. 18$)$, ou encore : «... le chaos n'étoit pas plus confus que les idées que M. De Luc a données sur les vapeurs dans ses recherches sur l'atmosphère» $\left({ }^{19}\right.$, p. $\left.45-46\right)$, et il enchaîne : «M. De Luc, quand il écrivoit ses recherches, n'avoit pas des idées plus nettes sur la différence qu'il y a entre la vapeur vésiculaire $\&$ la vapeur élastique» $\left({ }^{19}\right.$, p. 50$)$. Ce que personne aujourd'hui ne pourrait lui reprocher...

Aussi le choix de la chaux est un sujet de moquerie : «Pour obtenir ce terme [de sécheresse extrême], M. De Luc emploie la chaux à hautes doses; \& il ne lui a pas fallu un grand effort de génie pour substituer la chaux à l'alkali caustique que j'avois employé» $\left({ }^{19}\right.$, p. 28$)$.

Ce regrettable dissentiment entre les deux savants genevois fut encore porté sur le Journal de Paris. Saussure y écrira le 4. Septembre 1787, à propos de la précaution qu'il prit, sur la cîme du Mont-Blanc, d'enfermer son hygromètre dans une boîte humectée, avant l'observation, pour vérifier si le terme d'humidité absolue ainsi donné correspondait bien à celui obtenu sur la plaine (ce qui fut le cas) : «Je ferai voir dans peu combien les objections de M. De Luc contre cette manière d'obtenir l'humidité extrême sont mal fondées \& combien son nouvel Hygromètre est un instrument vicieux et trompeur» $\left({ }^{17}\right.$, p. 1076 , note).

La suite des événements montrera combien cette phrase blessa De Luc. Il y répondra, pourtant, très sereinement le 8 Octobre suivant, dans les Observations sur la Physique: «M. de Saussure se méprend; je n'ai rien dit contre cette manière de déterminer l'humidité extrême : je crois même qu'elle peut être bonne dans la construction de l'hygromètre de M. de Saussure, où le cheveu, alors lâche, peut s'appliquer contre les parois humectées de la boîte : ce qui revient à ma méthode, qui est de mouiller la substance hygroscopique : ce n'est donc pas sur cette opération que j'ai élevé des doutes, c'est sur la méthode fondamentale de M. de Saussure ; qui consiste à déterminer le point de l'humidité extrême sous une cloche qui repose sur l'eau \& dont les parois sont mouillées, sans avoir égard à la température : \& mon objection contre cette méthode, est le résultat de plusieurs expériences dans lesquelles j'ai trouvé, que sous cette cloche, l'humidité varioit avec la température. Je désire donc d'apprendre ce que M. de Saussure a trouvé de défectueux dans ces expériences, prêt à changer d'opinion si je me suis mépris » ( ${ }^{7}$, p. $377-378)$.

Mais Saussure revient sur la question dans le Journal de Paris du 4 Janvier 1788 : «Messieurs, c'est M. de Luc qui se trompe, quand il dit que le cheveu, alors lâche, 
pouvoit s'appliquer contre les parois humectées de la boîte, dans laquelle je plaçai mon hygromètre sur la cîme du Mont-Blanc. Mon hygromètre étoit suspendu au milieu de cette boîte; le contrepoids exerçoit librement son action sur le cheveu, \& celui-ci n'étoit ni lâche, ni appliqué contre les parois.» ${ }^{18}$

De Luc est alors excédé. Il écrit une nouvelle lettre le 19 Janvier 1788, publiée encore dans les Observations sur la Physique, où il laisse enfin éclater son indignation: "Je réponds encore à cette assertation avec le plus grand ménagement ; mais il m'arrive de faire une remarque (au fond indifférente) sur son opération hygrométrique, faite avant l'observation au Mont-Blanc, remarque à laquelle un fait, connu à lui mais non à moi, se trouvoit répondre; il me croit en faute \& il se hâte encore de réclamer contre cette remarque» $\left({ }^{8}\right.$, p. 139$)$, et son amertume : «... il [Saussure] saisit aux cheveux l'occasion qui lui fournissoient ses nouvelles observations hygrométriques aux Alpes, lieu qui auroit dû lui rappeler une grande réticence à mon égard, pour prononcer contre mon hygromètre, la sentence laconique [vicieux et trompeur] qui passe aujourd'hui de bouche en bouche dans toute l'Europe» $\left({ }^{8}\right.$, p. 139).

Pourtant, De Luc avait déjà tenté une manœuvre de réconciliation : «Frappé du ton que M. de Saussure prenoit si légèrement avec moi, quoique je n'y eusse jamais donné lieu, \& malgré des égards qu'il devoit sentir mieux que personne, je voulus tâcher de l'amener à une discussion plus paisible, en saisissant l'occasion de son voyage, pour lui donner de nouveau (non en flateur, mais en ami du vrai) les éloges que je lui crois dûs, \& en faisant mention de sa note [hygromètre de De Luc vicieux et trompeur], \& de quelques autres parties de sa relation (sur lesquelles je suis loin de lui donner des éloges) sans en montrer aucun ressentiment» $\left({ }^{8}\right.$, p. 134-135).

Cette manœuvre s'est donc avérée inutile. De Luc maintient alors ses positions et fait le point sur la cause réelle de leur désaccord : «... mais cette discussion [que le cheveu colle ou ne colle pas à la boîte humectée] est de fort petite importance. Ce qui importe de déterminer, est: 'si une cloche de verre, dont les parois intérieures sont mouillées \& qui repose sur l'eau, peut fournir un terme d'humidité qui soit le même à toute température'. C'est ce que M. de Saussure a conclu de ses expériences, mais dont j'ai trouvé le contraire par les miennes. Il y a donc quelque part des apparences qui méritent d'être observées de près. Et voilà à quoi je désire que M. de Saussure s'applique avec autant de sang-froid que je l'ai fait» $\left({ }^{8}\right.$, p. 137).

Il est difficile de savoir si, au moment où sont écrites ces lignes, De Luc avait déjà pris connaissance de la Défense de l'Hygromètre à cheveu de Saussure, qui ne fut portée au public, par les Observations sur la Physique, qu'en ce même mois de 
Janvier 1788. En tout cas, ses nombreux écrits, parus par la suite dans ce même journal, n'y feront jamais allusion. Il reparlera encore de l'hygromètre et des travaux de Saussure mais seulement pour les besoins du sujet exposé dans son texte et toujours avec la plus grande considération.

Presque deux siècles plus tard, Middleton jugera ainsi cette polémique entre les deux Genevois : "As far as Deluc was concerned this was grossly unfair, for in his scientific work he was as disinterested as his fellow Genevan. He stuck to his guns, and although time has proved De Saussure to have been right, Deluc clearly had the better temper" $\left({ }^{13}\right.$, p. 106).

Mais il trouve une excuse à Saussure: «His touchiness on this occasion may have been exaggerated by his bad health" $\left({ }^{13}\right.$, p. 106).

En effet, en Décembre 1785, Saussure démissionne, pour des raisons de santé, de la chaire de Philosophie de l'Académie de Genève qu'il occupait depuis 1762 (ce qui ne l'empêchera quand même pas de continuer ses chers voyages dans les Alpes...).

Quand à Jean Senebier, qui avait travaillé aussi en hygrométrie à la même époque et qui devait donc bien connaître la force des arguments en jeu, on le sent partagé par la réelle et égale estime qu'il portait aux deux antagonistes de cette dispute. A la mort de Saussure, en 1799, il écrira dans un livre consacré à sa mémoire :

«... il [Saussure] mit sans doute trop de chaleur dans sa 'Défense de l'hygromètre à cheveu' publiée en 1788 ; mais il faut reconnaître aussi qu'il a rendu trèssouvent à Deluc, avant et après cette controverse, la justice que ce grand physicien mérite à tant de titres» $\left({ }^{23}\right.$, p. 95$)$.

Le temps montra que Saussure se trompait, en qualifiant l'hygromètre à fanon de baleine de «vicieux et trompeur», ainsi que De Luc, en croyant que ce jugement passait « de bouche en bouche dans toute l'Europe». Les meilleurs fabricants d'instruments scientifiques européens, précisément, l'ont souvent construit et il fut même choisi, à cause de sa solidité, par Luke Howard pour ses observations sur le climat de Londres ${ }^{11}$. Mais ce fut la méthode de Saussure que Howard utilisa pour le calcul du point d'humidité extrême sur l'hygromètre de De Luc, méthode contre laquelle ce dernier s'éleva jadis.

En effet, il semble que, si le fait de plonger le fanon de baleine dans l'eau, ou dans un brouillard épais, donnait bien les $100^{\circ}$ de l'échelle de De Luc, le point d'humidité extrême de l'air ambiant ne correspondait qu'aux $80^{\circ}$ de cette échelle, d'où l'irrégularité des variations de l'hygromètre de De Luc sous la cloche de verre dès que le fanon entrait plus ou moins en contact avec l'eau qui se condensait autour. De Luc attribua ces variations aux changements de température, mais 
c'est finalement son système de mouillage de la substance hygroscopique qui en est le seul responsable.

Il sera peut-être intéressant d'ouvrir une petite parenthèse pour cerner, à la lumière de nos connaissances actuelles, les phénomènes assez délicats qui ont lieu dans un vase clos, tel que la cloche de verre de Saussure.

Il est certain que, quelque soit sa température, l'air enfermé dans cette cloche aura toujours une humidité relative égale à 1 (ou à $100 \%$, comme on l'exprime plus couramment) pour autant que la cloche soit constamment humectée. Par contre, son humidité absolue, c'est-à-dire, la quantité de vapeur d'eau qu'il contient, varie avec sa température car, plus cette température est élevée, plus il lui faut de vapeur d'eau pour atteindre le point de saturation. Le Roy l'avait déjà expliqué clairement en 1751 et De Luc, aussi bien que Saussure, étaient très au courant de la théorie du médecin de Montpellier.

De Luc dira même de lui : «Si cette partie de la Physique [l'hygrométrie] s'éclaircit un jour, comme je l'espère, elle devra beaucoup à la sagacité de ce vrai Physicien» $\left({ }^{5}\right.$, p. 474$)$.

Et Saussure, à son tour : «... les belles expériences de M. Le Roi ont fait voir que l'air est un vrai dissolvant des vapeurs, \& que la chaleur augmente sa force dissolvante. S'il pouvoit en rester quelque doute sur cette vérité, l'Hygromètre en fourniroit la preuve» $\left({ }^{16}, \S 20\right)$.

Mais pour tout le monde à cette époque, l'humidité continue à être une notion unique et absolue, la quantité de vapeur d'eau dans un certain volume d'air.

Ainsi, nous pouvons presque nous étonner que la méconnaissance même du phénomène qui fait marcher la substance hygroscopique sur laquelle étaient basés les hygromètres d'alors, et que nous traduisons aujourd'hui par humidité relative, n'ait pas engendré de plus grandes confusions parmi les physiciens qui l'étudiaient. Et si à cette importante lacune nous ajoutons encore les difficultés que posent les fréquentes irrégularités des variations de la substance hygroscopique, nous pouvons mieux apprécier la qualité du travail des deux savants genevois. Leur dissentiment porte surtout sur des questions techniques. Mouillage intégral ou saturation sous cloche de verre, le choix au premier abord semble quand même empirique et mériterait d'être discuté d'après les résultats des expériences. De Luc eut le tort de trancher trop vite et d'opter, évidemment, pour sa méthode. Quant il a voulu l'analyser conjointement avec Saussure, ce dernier, sûr des avantages de la sienne, refusa alors tout dialogue.

La violence que Saussure a donné à cette polémique et la profondeur de son impact sur De Luc laissent supposer que l'affaire se jouait autant sur le plan personnel que sur le plan scientifique. Mais essayons de rester sur ce dernier. 
L'idée d'humidité relative finira par germer quelques années plus tard et ce sera encore De Luc qui y pensera le premier. En effet, il avait écrit, en 1792, que : «... si ... on cherche à connaître la densité de cette vapeur d'eau, ou sa quantité dans un espace donné, l'observation de la température est nécessaire : car cela, par des expériences préalables en vue de déterminer les quantités de vapeur correspondantes à ses différents maximums selon la température, procurera un coefficient pour la fraction observée» $\left({ }^{9}\right.$, p. $413-414$, trad. $)$.

Mais c'est Gilbert qui, en 1803, la définit clairement: «Le degré d'humidité dépend de la raison entre la vapeur d'eau présente à ce moment-là et celle qu'il serait possible» $\left({ }^{10}\right.$, p. 167 , trad.).

Maintenant que toutes les données sont formulées, on voit apparaître, vers 1820 , le premier hygromètre à condensation inventé par Daniell ${ }^{2}$. Reposant directement sur la théorie, cet appareil ne pouvait connaître par la suite que des perfectionnements d'ordre pratique, dont notamment ceux qui lui furent apportés par Regnault, Alluard et Crova tout au long du XIX ${ }^{\mathrm{e}}$ siècle et qui ont fait de lui, encore maintenant, l'hygromètre de précision par excellence.

L'hygromètre à cheveu de Saussure survivra, pourtant, à l'invention de l'hygromètre à condensation de Daniell et fera même une carrière scientifique importante jusqu'en 1845, date à laquelle les expériences de Regnault sur plusieurs de ces hygromètres à cheveu, dont un fabriqué par Paul à Genève, ont démontré qu'ils «... ne marchent pas rigoureusement d'accord, mais que cependant ils ne s'éloignent pas assez pour que, dans la plupart des observations, on ne puisse les regarder comme comparables» $\left({ }^{14}, \mathrm{p} .171\right)$.

Dès lors, l'emploi des hygromètres munis d'une substance hygroscopique, qu'on appelle aussi hygromètres hygroscopiques et dont le plus renommé était sans aucun doute celui de Saussure, fut relégué à un rôle de second plan.

Nous pouvons donc résumer l'apport genevois à l'Hygrométrie en faisant remarquer que ce furent De Luc et, surtout, Saussure qui portèrent l'hygromètre hygroscopique à un si haut niveau de perfection que, pour faire mieux, il a fallu choisir une autre formule, celle de l'hygromètre à condensation.

De Luc contribua encore à cette transition en reconnaissant, le premier, que l'humidité observée devait s'exprimer par une fraction.

Et même Senebier, qu'il ait ou non influencé Saussure, a le mérite d'avoir trouvé, par ses propres moyens, la bonne voie pour le calcul des points d'humidité et de sécheresse extrêmes. 


\section{Bibliographie}

1 Archinard, Margarida, De Luc et la recherche barométrique. Gesnerus 32 (1975), p. 235-247.

2 Daniell, John Frederick, Meteorological essays and observations, London 1823.

${ }^{3}$ Daumas, Maurice, Les instruments scientifiques aux XVII ${ }^{e}$ et $X V I I I^{e}$ siècles, Paris 1953.

${ }^{4}$ De Luc, Jean André, Recherches sur les modifications de l'Atmosphère, 2 vols., Genève $1772 ; 2^{\mathrm{e}}$ édition, 4 vols., Paris 1784.

5 - Copie d'un mémoire sur un hygromètre comparable. Observations sur la Physique 5 (1775), p. 381-476.

${ }^{6}$ - Idées sur la Météorologie, 2 vols., $8^{\circ}$, Londres 1786 ; et une $2^{\mathrm{e}}$ édition presque identique, Paris 1787.

7 - Lettre sur les observations faites par M. de Saussure sur la cîme du Mont-Blanc. Observations sur la Physique 31 (1787), p. 374-381.

8 - Lettre à M. De La Métherie sur l'hygromètre de baleine. Observations sur la Physique 32 (1788), p. 132-140.

9 - On evaporation. Philosophical Transactions 82 (1792), p. 400-424.

10 Gilbert, Ludwig Wilhelm, Einige Bemerkungen zu Dalton's Untersuchungen über die Verdünstung. Annalen der Physik 15 (1803), p. 144-168.

11 Howard, Luke, The Climate of London, 2 vols., London 1818-1820.

${ }^{12}$ Le Roy, Charles, Mémoire sur l'élévation et la suspension de l'eau dans l'air et sur la rosée. Mémoires de l'Académie Royale des Sciences de Paris (1751), p. 481-518.

13 Middleton, W. E. Knowles, Invention of the Meteorological Instruments, Baltimore 1969.

14 Regnault, Victor, Etudes sur l'hygrométrie. Annales de Chimie et de Physique 15 (1845), p. 129-236.

15 Saussure, Horace Bénédict de, Lettre à M. Senebier. Observations sur la Physique 2 (1778), p. 435-439.

16 - Essais sur l'Hygrométrie, Neuchâtel 1783; 2 éditions presque identiques dans la même année, $4^{\circ}$ et $8^{\circ}$.

17 - Notice des observations faites sur la cîme du Mont-Blanc le 3 Août 1787. Journal de Paris, 4. Septembre 1787, p. 1075-1077.

18 - Lettre aux Auteurs du Journal. Journal de Paris, 4 Janvier 1788, p. 18.

19 - Défense de l'hygromètre à cheveu, Genève 1788.

20 - Description d'un cyanomètre, ou d'un appareil destiné à mesurer l'intensité de la couleur bleue du ciel. Mémoires de l'Académie des Sciences de Turin (1788-89), p. 409-424; Observations sur la Physique 38 (1791), p. 199-208.

21 - Description d'un diaphanomètre, ou d'un appareil propre à mesurer la transparence de l'air. Mémoires de l'Académie des Sciences de Turin (1788-89), p. 425-440.

22 Senebier, Jean, Premier mémoire sur les hygromètres. Observations sur la Physique 2 (1778), p. 421-435.

23 - Mémoire historique sur la vie et les écrits de Horace Bénédict Desaussure, Genève, l'an IX de la Révolution Française. 


\section{Summary}

Three Geneva scientists contributed to the development of hygrometry: J.A. De Luc through his hygrometers (the first made of ivory, the last in whalebone), his judicious choice of two fixed points in graduating them, his prevision of a fraction to express the humidity observed; J. Senebier through his procedures to determine the fixed points envisaged by De Luc; H. B. de Saussure with his famous hair hygrometer, his improvements of the procedures of Senebier (has he found them alone?), his tables and conclusions, which have brought about the use of his apparatus till the middle of the 19th century. A regrettable polemic between de Saussure and De Luc developed around their respective hygrometers.

Dr. math. Margarida Archinard 1 , chemin de l'Escalade

1206 Genève 